\title{
Cidades Inteligentes: Uma Arquitetura de Referência para Sistemas de Informação Baseada na Perspectiva Social
}

\author{
Alexandre Pires Barbosa ${ }^{1}$, Marcelo Fornazin (colaborador) ${ }^{2}$, \\ Rodrigo Pereira dos Santos (orientador) ${ }^{1}$
}

\author{
${ }^{1}$ Programa de Pós-Graduação em Informática (PPGI) \\ Universidade Federal do Estado do Rio de Janeiro (UNIRIO) \\ Avenida Pasteur, 458, Urca - Rio de Janeiro - RJ - Brasil
}

${ }^{2}$ Departamento de Ciência da Computação - Universidade Federal Fluminense (UFF) Avenida General Milton Tavares de Souza, s/n, São Domingos - Niterói - RJ - Brasil

alexandre.barbosa@edu.unirio.br, fornazin@ic.uff.br, rps@uniriotec.br

\begin{abstract}
Promoting sustainable and more equal actions to orderly urban growth is a challenge that smart cities aim to solve. Information systems play a fundamental role in facing the challenges of urban space management, as their approaches include the involvement of people, organizations and technologies. However, such approaches can generate conflicts of interest when they disregard citizens in their goals. This research project aims to investigate possibilities and challenges for smart cities and to propose a reference architecture to guide researchers and governments in a social approach for smart cities.
\end{abstract}

Resumo. Promover ações sustentáveis e mais igualitárias voltadas para o crescimento urbano ordenado é um desafio que as cidades inteligentes visam resolver. Os sistemas de informação têm papel fundamental para enfrentamento dos desafios da gestão do espaço urbano, pois suas abordagens compreendem o envolvimento de pessoas, organizações e tecnologias. Contudo, estas abordagens podem gerar conflitos de interesses quando desconsideram os cidadãos em seus objetivos. Este projeto de pesquisa visa investigar possibilidades e limites para as cidades inteligentes e propor uma arquitetura de referência para guiar pesquisadores e governos em uma abordagem social para cidades inteligentes.

\section{Introdução}

Desde a revolução industrial, a humanidade vem sofrendo mudanças significativas, nas quais empresas, governos e sociedade vêm sendo constantemente desafiadas a superar os desafios impostos por esta nova realidade [Zhou et al. 2015]. No contexto das cidades, que irá concentrar $70 \%$ da população mundial, há uma expectativa de que, com o aumento populacional previsto até 2050, surjam consequências drásticas para a sociedade quando não ordenado. Isso se refletirá ainda mais no aumento de problemas urbanos, como níveis de pobreza, violência, desigualdades sociais e aquecimento global que resulta no desequilíbrio ambiental [Soupizet 2017].

As cidades inteligentes, do inglês smart cities, se apresentam como uma proposta para oferecer melhores serviços públicos utilizando tecnologias digitais para prover melhor qualidade de vida para os cidadãos. Entretanto, por se tratar de um conceito complexo 
que envolve interesses distintos, a sua definição ainda não foi consolidada por pesquisadores e reconhecida pelos cidadãos, principalmente devido à existência de dimensões estruturais e múltiplos objetivos a serem alcançados [Nam e Pardo 2011].

A área de Sistemas de Informação (SI) se apresenta com papel fundamental para dar suporte às cidades inteligentes, no sentido de reduzir os impactos sociais, ambientais e econômicos, visto que uma de suas principais abordagens se relacionam aos objetivos de governança. Porém, devido à esta área contemplar três aspectos distintos, como pessoas, processos e tecnologias, existem conflitos de interesses que precisam ser mapeados e mitigados para implantação dos projetos de cidades inteligentes.

Neste cenário, as questões sociais são pouco exploradas na literatura e podem enfraquecer os projetos de cidades inteligentes, devido à heterogeneidade de pessoas, desigualdades sociais, variação de renda e aspectos culturais que estão inseridos nas cidades. A Organização das Nações Unidas (ONU), por meio dos Objetivos de Desenvolvimento Sustentável (ODS), também vem trabalhando em ações que envolvem fatores sociais, no sentido de oferecer condições igualitárias e sustentáveis a todos os cidadãos [Desa et al. 2016]. O objetivo da pesquisa consiste em investigar e compreender os fatores sociais aplicados às cidades inteligentes no sentido de criar uma arquitetura de referência para SI neste contexto. A meta é auxiliar pesquisadores, governos e desenvolvedores de soluções para cidades inteligentes com uma proposta para considerar fatores sociais reconhecidos pelos cidadãos na implantação dos projetos.

\section{Apresentação do Problema}

Sendo um assunto amplamente discutido na literatura, cidades inteligentes podem possuir diversos conceitos e a sua definição pode ser apresentada envolvendo a utilização de tecnologias digitais para gestão urbana [Zanella et al. 2014]. Segundo Nam e Pardo (2011), estas definições também podem ser abordadas considerando meios subjetivos voltados para qualidade de vida e desempenho futuro das cidades.

Além destas questões, as cidades inteligentes são representadas em dimensões complexas, utilizando a classificação de serviços oferecidos pelos governos, como saúde, mobilidade, segurança, educação, ou sobre outra visão, considerando aspectos mais abrangentes, como economia, meio ambiente, infraestrutura e tecnologia [Albino e Dangelico 2015]. A ONU também endereça as questões urbanas por meio dos ODS com vistas a oferecer aos cidadãos qualidade de vida em condições igualitárias. Diante deste aspecto, existe uma forte atuação entre 197 países dedicados a trabalhar nos fatores sociais, econômicos e ambientais presentes nas cidades, com objetivo de garantir o desenvolvimento sustentável da população [Desa et al. 2016].

A variedade de abordagens, definições e conceitos são apresentados considerando características de infraestruturas tecnológicas, utilizando o potencial das tecnologias da informação e comunicação (TIC) para resolver problemas urbanos e com pouca ênfase nos fatores sociais [Caragliu et al. 2009]. Sendo assim, faz-se necessário elaborar uma conceituação de forma que os SI sejam utilizados estrategicamente para atender os interesses dos stakeholders, envolvendo fatores sociais que geram valores reconhecidos pelos cidadãos e promovendo o desenvolvimento sustentável. 


\section{Projeto da Pesquisa}

Para solucionar o conflito de entendimentos, abordagens e interesses na implantação das cidades inteligentes, a proposta deste trabalho consiste em elaborar uma arquitetura de referência para SI no domínio de cidades inteligentes, considerando os fatores sociais descritos nos ODS. Arquitetura de referência é definida como um conceito mais amplo da arquitetura de software, em maior nível de abstração, considerando aspectos de qualidade, melhores práticas e relacionamentos e, inclusive, o tripé da área de SI, que corresponde a pessoas, processos/organizações e tecnologias [Bass et al. 2003].

Sabe-se que os SI possuem características fundamentais, que são capazes de coletar, recuperar, processar, armazenar e distribuir informações para gerir a complexidade de grandes organizações e centros urbanos [Laudon e Laudon 2000]. A elaboração de uma arquitetura de referência para SI no domínio de cidades inteligentes poderá oferecer uma melhor compreensão do todo, visto que a utilização dos SI pela administração pública se diferencia do setor privado por ir além da eficiência e eficácia dos recursos, que inclui o fomento às políticas sociais, como inclusão, resiliência da comunidade, qualidade de vida e sustentabilidade [Grimsley e Meehan 2007].

As arquiteturas de referências desempenham um papel fundamental no entendimento dos domínios em que estão aplicados, pois permitem capturar a essência dos projetos e os interesses dos stakeholders, encapsulando conhecimento no sentido de disseminálo e reutilizá-lo no desenvolvimento de sistemas [Nakagawa et al. 2014]. O principal fator de sucesso para utilização das arquiteturas de referência consiste na abordagem sistêmica, pois ela nasce da evolução da arquitetura de software, considerando visões de arquitetura de dados, arquitetura voltada para negócios e arquitetura de infraestrutura tecnológica [Domingos 2004].

Diante da motivação desta abordagem, para atingir o objetivo da pesquisa, o estudo irá se fundamentar em uma metodologia que envolve três etapas conforme detalhamento na Figura 1. A primeira etapa consiste na Concepção, a segunda etapa visa a Implementação e, por fim, a terceira foca na Avaliação. Estas etapas estão detalhadas nas Seções 4 e 5.

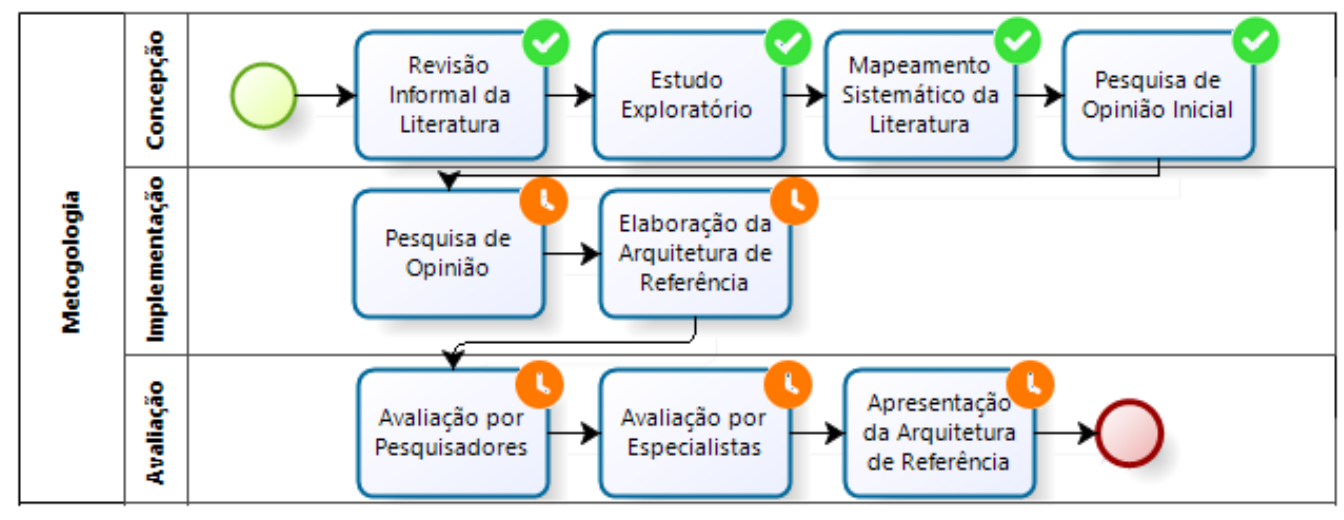

Figura 1. Metodologia

\section{Projeto de Avaliação}

O projeto de elaboração da arquitetura de referência para SI no domínio de cidades inteligentes será avaliado em duas etapas, que correspondem às etapas de implementação 
e avaliação da Figura 1. A primeira ocorrerá por meio do diálogo com cidadãos, a fim de compreender as necessidades regionais e tecnologicas, considerando também os fatores demográficos, sociais e tecnológicos, as questões de acesso e a utilização de serviços digitais oferecidos pela administração pública e pela iniciativa privada.

A segunda etapa visa avaliar a utilização da proposta de arquitetura de referência para SI por pesquisadores e especialistas em cidades inteligentes, no sentido estruturar os sistemas que dão suporte às cidades inteligentes. Nesta etapa, o processo de avaliação irá considerar um padrão arquitetural que irá expressar a relação entre contexto, problema e uma solução. Desta forma, a arquitetura será avaliada considerando: a) o contexto de utilização, b) objetivos estratégicos e, c) detalhes do técnicos do projeto [Silva e Nakagawa 2013].

\section{Atividades Realizadas}

As atividades realizadas nesta pesquisa correspondem àquelas descritas na etapa de concepção da Figura 1. Inicialmente, realizou-se a leitura de artigos relacionados ao tema de cidades inteligentes. Nesta etapa, foi possível entender as abordagens deste projeto, sobressaindo mais projetos técnicos e menos abordagens sociais. Também foi realizado um estudo exploratório que teve como objetivo investigar oportunidades e barreiras no desenvolvimento de sistemas informais no domínio de cidades inteligentes, além de utilizar procedimentos de Grounded Theory para analisar a opinião dos cidadãos que participaram de um estudo exploratório e discutir os resultados diante do estado da arte. Como conclusão, foi possível compreender as principais necessidades de uma cidade sob o ponto de vista do cidadão, bem como as principais barreiras que dificultam o desenvolvimento de SI para apoiar este contexto.

Além disso, foi conduzido um mapeamento sistemático da literatura (MSL) com o objetivo de identificar os principais SI no domínio governamental. Como conclusão, foram identificados 20 sistemas. Entre os mais citados, de acordo com os autores dos estudos analisados, os sistemas de informações geográficas foram capazes de reunir dados e informações sobre energia, otimização de trânsito, geolocalização de agências de serviços públicos, coleta de recicláveis, surgimento de favelas, desastres naturais e integração de mídias sociais. Por fim, foi realizada uma pesquisa de opinião inicial com objetivo de avaliar a utilização dos principais SI no domínio governamental no contexto brasileiro, que foram identificados no MSL, além de buscar a opinião dos respondentes sobre fatores de sucesso e desafios para implantação de SI em cidades inteligentes.

\section{Considerações Finais}

Este trabalho apresenta um projeto de pesquisa de dissertação que visa lançar luz sobre as abordagens de cidades inteligentes baseada em uma perspectiva social, considerando os ODS para criação de uma arquitetura de referência para SI. Como contribuições do trabalho, estão previstas uma síntese de estudos de literatura de cidades inteligentes sob os fatores sociais e uma análise de como os SI poderão auxiliar os projetos considerando os cidadãos, além de apoiar pesquisadores, governos e especialistas no entendimento de cidades inteligentes que geram valor e são reconhecidos pelos cidadãos. A sua relevância se apresenta devido à organização do conhecimento alinhado aos ODS, utilizando SI em cidades inteligentes sob uma perspectiva social. 


\section{Agradecimentos}

Os autores agradecem a UNIRIO e FAPERJ (Proc. 211.583/2019) pelo apoio à pesquisa e a Paulo Malcher (doutorando do PPGI/UNIRIO) pela parceria nesta pesquisa.

\section{Referências}

Albino, V. e Dangelico, R. (2015). Smart cities: Definitions, dimensions, performance, and initiatives. Journal of Urban Technology, 22:3-21.

Bass, L., Clements, P. e Kazman, R. (2003). Software architecture in practice. AddisonWesley Professional.

Caragliu, A., Del Bo, C. e Nijkamp, P. (2009). Smart cities in europe. VU University Amsterdam, Faculty of Economics, Business Administration and Econometrics, Serie Research Memoranda, 18.

Desa, U. et al. (2016). Transforming our world: The 2030 agenda for sustainable development.

Domingos, M. (2004). Uma arquitetura de referência para sistemas de informação e portais de serviços de governo eletrônico.

Grimsley, M. e Meehan, A. (2007). e-government information systems: Evaluation-led design for public value and client trust. European Journal of Information Systems, 16(2):134-148.

Laudon, K. C. e Laudon, J. P. (2000). Management information systems: organization and technology in the networked enterprise. Prentice Hall International.

Nakagawa, E. Y., Guessi, M., Maldonado, J. C., Feitosa, D. e Oquendo, F. (2014). Consolidating a process for the design, representation, and evaluation of reference architectures. In 2014 IEEE/IFIP Conference on Software Architecture, pages 143-152. IEEE.

Nam, T. e Pardo, T. A. (2011). Conceptualizing smart city with dimensions of technology, people, and institutions. In Proceedings of the 12th annual international digital government research conference: digital government innovation in challenging times, pages 282-291.

Silva, J. e Nakagawa, E. (2013). Um processo para avaliação de arquiteturas de referência.

Soupizet, J. F. (2017). Cidades inteligentes: desafios para as sociedades democráticas. Rio de Janeiro: Centro Edelstein de Pesquisas Sociais.

Zanella, A., Bui, N., Castellani, A., Vangelista, L. e Zorzi, M. (2014). Internet of things for smart cities. IEEE Internet of Things journal, 1(1):22-32.

Zhou, K., Liu, T. e Zhou, L. (2015). Industry 4.0: Towards future industrial opportunities and challenges. In 2015 12th International conference on fuzzy systems and knowledge discovery (FSKD), pages 2147-2152. IEEE. 